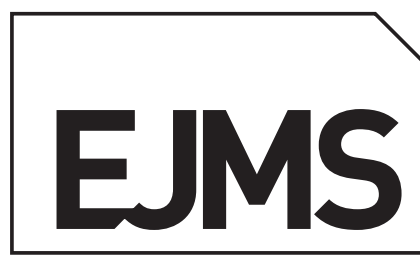

EUROPEAN

JOURNAL

OF

MASS

SPECTROMETRY

Proceedings of the $18^{\text {th }}$ International Conference on Mass Spectrometry

\title{
Electrochemical reactions and ionization processes
}

\author{
Hubert Girault, ${ }^{\mathrm{a}}$ BaoHong Liu, ${ }^{\mathrm{b}}$ Liang Qiao, ${ }^{\mathrm{a}, \mathrm{b}}$ HongYan Bi, ${ }^{a}$ Michel Prudent, ${ }^{a}$ Niels Lion ${ }^{\mathrm{a}}$ and Mélanie Abonnenc ${ }^{\mathrm{a}}$ \\ aLaboratoire d'Electrochimie Physique et Analytique, Ecole Polytechnique Fédérale de Lausanne, Station 6, CH-1015 Lausanne, Switzerland. \\ E-mail: hubert.girault@epfl.ch \\ ${ }^{b}$ Department of Chemistry, Institutes of Biomedical Sciences, Fudan University, Shanghai 200433, PR China
}

\begin{abstract}
Electrochemical or photo-electrochemical reactions in both electrospray ionization and laser desorption ionization are discussed stressing the role of the electrode reaction in influencing the ionization process. In particular, upon application of a high voltage during electrospray ionization, the emitter includes a working electrode, where redox reactions are observed, such as electro-generation of benzoquinone and metal ions. In contrast, the target plate in laser-induced desorption ionization also acts as a photo-electrode, especially when modified with a mesoporous semiconductor. We illustrate here how these electrochemical reactions can be used for tagging purposes and for oxidative or reductive dissociation reactions.
\end{abstract}

Keywords: electrospray ionization, emitters, LDI, titanium dioxide nanoparticle, semiconductor, peptide in-source-decay, cysteine tagging, proteomics

\section{Introduction}

During the last decades, electrospray ionization (ESI) ${ }^{1}$ and matrixassisted laser desorption/ionization (MALDI) ${ }^{2}$ have been developed as standard soft ion sources for the mass spectrometry analysis of big molecules, such as polymers and biomolecules. Indeed, the rapid development of proteomics stems mostly from the success of these two techniques. ${ }^{3}$ Compared to the biological applications, the fundamental mechanistic aspects of the ionizations methods have received much less interest. ${ }^{4}$ However, the understanding of the basic principles are, of course, very important and can be helpful for developing new techniques.

In this short review, we mainly focus on some work performed in our laboratories regarding the electro-chemical aspects of electrospray and photo-electrochemical aspects of laser desorption/ionization (LDI). In ESI, a high voltage is applied to a sample solution located within a capillary. The electrode contacting the solution is the locus of electrochemical reactions that can be put to good use, thereby adding functionalities to the spray process.

If the electrochemical aspects of ESI are by now well established, those of LDI have only rather recently become a topic of interest. Different from electrospray, the electrode reaction during LDI is not often recognized, as the high voltage is applied with a delay after the laser pulse. Indeed, delayed extraction is widely used for time-of-flight (TOF) analyzer. However, electrochemical reactions that are induced by photoelectrons generated from the metallic substrate during MALDI and by electron/hole pairs generated in the semiconductor structure during desorption/ionization on silicon (DIOS) ${ }^{5}$ or $\mathrm{TiO}_{2}$ have often been observed. Besides, it is believed that electrode reactions must occur on the target plate as soon as the extraction voltage is applied to ensure the continuity of the current, while the results of this electrode reaction may influence the subsequent laser shots.

As an example of useful electrochemical reactions, electrogeneration of benzoquinone was performed with both ESI and LDI for on-line tagging cysteine-containing peptides; electrogeneration of metal ions during ESI was used to study the on-line peptide complexation with transition-metal ions; photoelectrode assisted LDI in-source oxidative peptide dissociation 


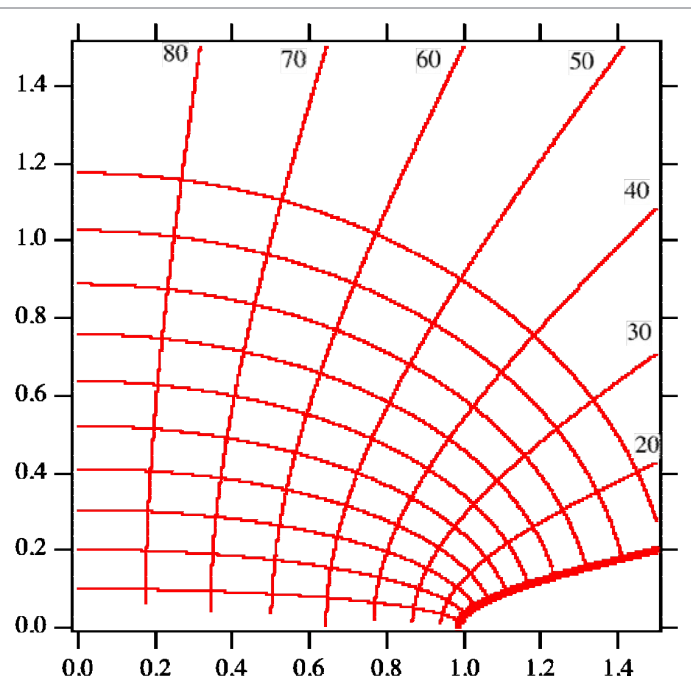

Figure 1. Electric field distribution at an electrified point. Half a point is represented by a bold line at the bottom left of the graph. The equipotential line at $x=0$ represents a planar counter-electrode. The lines of current are represented by the ellipses and the equipotential by the hyperbola.

generating $a, x$-fragments was observed along with the reductive peptide dissociation producing $c, z$-fragments. All these applications are useful in view of proteomics; and with these successes, further investigation should be promoted.

\section{Electrochemical aspects of electrospray ionization}

Electrospray is a phenomenon that was studied as early as 1749 when Nollet described the spray from a metallic orifice that was electrified electrostatically, ${ }^{6}$ but it was only at the beginning of the $X X^{\text {th }}$ century that Zeleny pictured the process in which a liquid is drawn to form a fine mist of droplets. ${ }^{7}$ In 1964, Taylor showed that the shape of the electrolyte/ air interface under the application of a potential difference between an electrode in solution and an electrode located outside and facing the orifice sustaining the interface tends towards a conical shape, now referred to as the Taylor cone. ${ }^{8}$ In 1928, Eyring et al. had shown that if the shape of the cone can be assumed to be a hyperboloid, ${ }^{9}$ then the equipotential lines are hyperbola as shown in Figure 1.

From an electrochemical standpoint, the charging of the electrolyte/air interface results in a potential distribution within the cone that can be described by the Gouy-Chapman model of diffuse double layers. In other words, the potential drop in the solution side will be less than one volt. The relationship between the charge density, $\Sigma$, on the aqueous side of the interface and the electric field, $E$, just outside the cone is given by Gauss's law, i.e.

$$
E=\frac{\sigma}{\varepsilon_{0}} \hat{n}
$$

where $\hat{n}$ represents the unit normal vector to the surface and $\varepsilon_{0}$ the vacuum permittivity.

As we can see from Figure 1, the electric field is strongest at the tip. Since according to Lippmann's electrocapillary theory, the surface tension decreases as the surface charge density increases, the surface tension holding the cone is lowest at the tip and this is why when the electric field reaches a critical value, then the interface breaks at the tip and small droplets are emitted. Figure 2 shows the effect of an electric field in the shape of a pendant drop and how it tends from a classical pendant drop to a conical shape.

The electrochemical aspects of a charged droplet are also interesting and worth discussing. In the field of electrochemistry at liquid-liquid interfaces, Marcus proposed that ion transfer

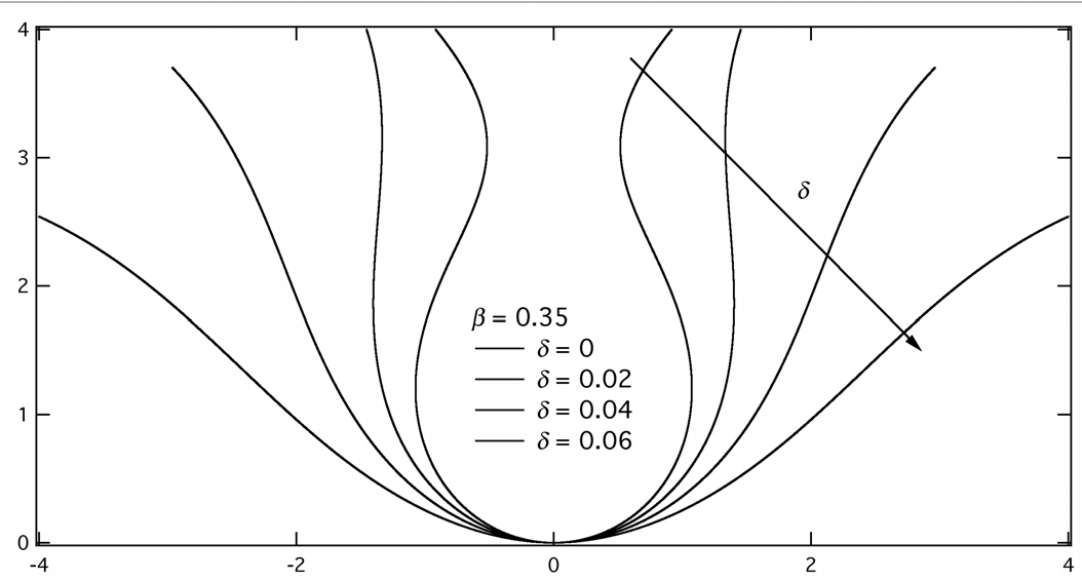

Figure 2. Pendant drop shape upon application of an electric field. $\beta$ and $\delta$ are dimensionless parameters $\beta=\rho g b^{2} / y$ and $\delta=\sigma E b / \gamma$ where $\rho$ is the volumic mass of the solvent, $\sigma$ the surface charge density, $g$ the gravity acceleration, $E$ the electric field assumed constant at the surface of the droplet, $b$ the apex radius of curvature and $\gamma$ the interfacial tension. 


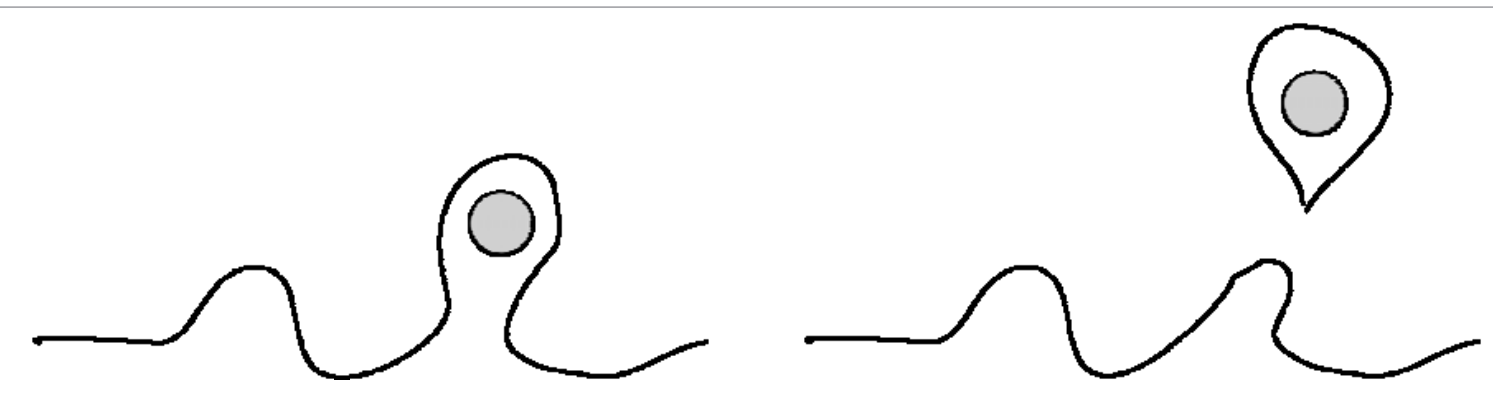

Figure 3. Marcus ion transfer model where ion transfer reactions from a bottom aqueous layer to an upper organic phase occurs via the interface motion (for example, capillary wave) where an aqueous protrusion containing an ion is formed and then detaches from the interface.

reactions, say from an aqueous phase to an organic phase, occurs through the formation of "hernia" formed during the capillary motion occurring at the interface, as depicted in Figure 3. ${ }^{10}$ This mechanism is analogous to the ion evaporation model proposed by Iribarne and Thomson. ${ }^{11}$ However, Marcus's model suggests that ion evaporation is more a consequence of the interfacial dynamics than a direct consequence of electrostatic repulsion within the Gouy-Chapman diffuse layer. Of course, as soon as a hernia is formed, then electrostatic repulsion is the driving force to repel the ion from the droplet.
Regarding the solution side, the electrode applying the potential difference with respect to the gas phase is, by definition, in contact with the electrolyte solution. In positive ion mode, this electrode acts as an anode and is, therefore, the locus of oxidation reactions, as illustrated in Figure 4(a). Conversely, in negative ion mode, the electrode acts as a cathode and is therefore the locus of reduction reactions.

If we apply a potential drop, $\Delta \mathrm{V}$, between the electrode in solution and the mass spectrometer acting as a counter electrode in the gas phase, it is important to realize that almost all

(a)

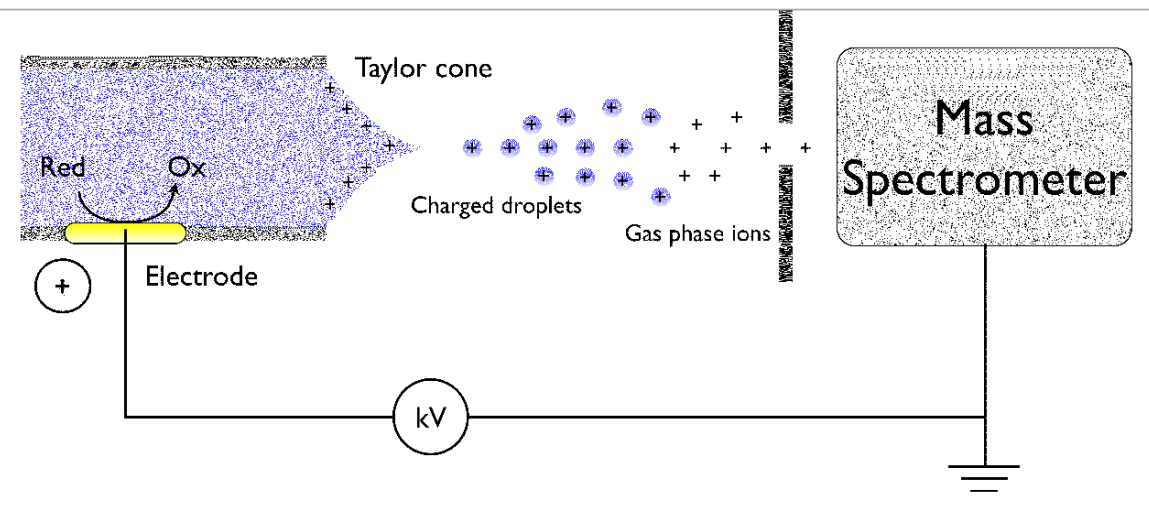

(b)

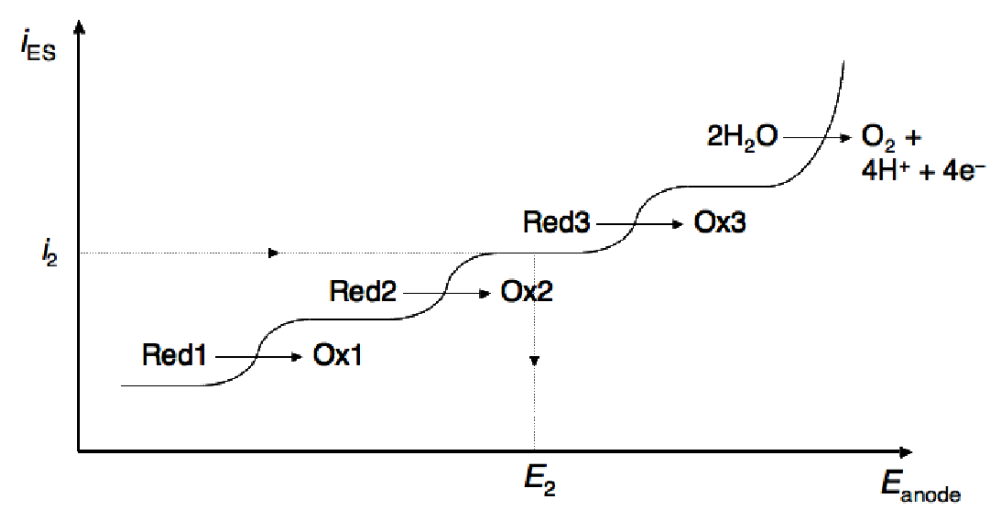

Figure 4. (a) Schematic aspects of electrospray ionization. (b) Schematic representation of the current-potential curve. 
the potential drops occur in the gas phase. According to Ohm's law, the resistance between the two electrodes is the sum of the resistance in the solution and in the gas phase, the former being negligible compared to the latter. Therefore, from an electrochemical standpoint, an ESI operates a two-electrode cell, where the gas phase resistance limits the current in normal operating conditions to sub-microamp levels. As a result, the potential drop at the electrode is, at most, one or two volts, which, in solution, is negligible and, at the solution/ air interface, is no more than one volt.

The electrochemical reactions taking place at the electrode will depend on the concentrations of the different redox species and on the kinetics of the charge transfer reactions. If we assume a steady state current, the oxidation current can be described as the sum of the different oxidation processes, the species with the lowest redox potentials being oxidized first until the solvent becomes oxidized. In the latter case, the oxidation of water will produce oxygen and protons. It is important to realize that the electrode reaction operates at constant current, as discussed above, and that the electrode potential will adapt to provide this current, as shown in Figure 4(b).

\section{Electrochemically-initiated homogeneous reaction}

\section{Electrogeneration of benzoquinone tags from hydroquinones}

Over the last years, electrochemically-initiated homogeneous reactions, directly in the electrospray emitter, were extensively studied in our lab. One major investigation concerns the electrogeneration of benzoquinone tags that react specifically with thiol moiety of cysteines according to a 1,4-Michael addition, under acidic conditions. A novel methodology based on electrochemical tagging of cysteines, by in-situ oxidation of hydroquinone into benzoquinone at the electrode, during ESI-MS of peptides has been introduced. ${ }^{12-14}$ The potential of this on-line electrochemical tagging was demonstrated with microfabricated electrospray emitters, where instrumental parameters (current density at the electrospray electrode, residence time of analytes and chemical probes...) can be properly tailored and controlled. The influence of the tagging rate constant as well as the impact of the probe and target concentrations was investigated numerically for single and consecutive tagging reactions in microchannels. ${ }^{15}$ This method has been successfully used to count on-line the cysteine residues in tryptic peptides during ESI-MS, which is relevant information to definitively ensure the protein identification in peptide mass fingerprinting analysis. ${ }^{16}$ However, this methodology has so far been limited to off-line analysis of peptide fractions and nano-flow rates. In order to bring the potential of tagging methodologies to on-line postcolumn derivatization (for example, capillary high-performance liquid chromatography-ESI-MS analysis) of tryptic peptides, we have developed an electrospray micromixer chip where the sample is physically mixed with labeling reagent. ${ }^{17}$ This device was demonstrated to be an efficient tool for on-line derivatization of biomolecules and kinetics studies. Last, but not least, the electrochemical tagging of the cysteine residues was demonstrated as a useful tool to probe their environment. ${ }^{18}$ The effect of the cysteine site reactivity on the tagging efficiencies was highlighted when comparing unmodified proteins and their chemically reduced forms.

\section{Electrogeneration of metal ions from sacrificial electrodes}

Another line of investigation concerns the study of bioinorganic compounds by electrospray mass spectrometry relying on the concept of sacrificial electrodes. Soluble anodes, i.e. electrodes made of a metal or alloy that dissolves anodically at low potentials, such as copper, iron, or even silver, have been used to study the on-line peptide complexation with transitionmetal ions. ${ }^{19}$ In the case of copper, our group has shown that during the anodic dissolution process Cu(I) is formed. In the presence of specific ligands, Cu(I) complexes can then be generated in solution and analyzed by MS. Otherwise, Cu(I) ions are further oxidized to Cu(II), which enables the study of complexation sites of metal ions with different degree of oxidation. ${ }^{20}$ This method was applied to the on-line electrogeneration of copper-peptide complexes in an electrospray emitter microchip. ${ }^{21}$ The concept of sacrificial electrodes was successfully applied to the tagging of phosphopeptides in a dual-channel emitter that consists of two different channels providing the phosphopeptides and the tag, respectively, which are only in contact at the emitter tip, i.e. at the Taylor cone. ${ }^{22}$ In this latter application, a zinc electrode was used to in-situ electrogenerate the dinuclear zinc(II) tag, which can, in turn, react with phosphate groups in peptides at the Taylor cone.

\section{Biphasic electrospray to study interfacial complexation reactions}

Biphasic electrospray was introduced for the study of interfacial complexation reactions using a dual-channel electrospray emitter chip, where the two solutions meet at the Taylor cone. Both the interfacial complexation of aqueous lead ions by thioether crown molecules and the complexation of an aqueous dipeptide with dibenzo-18-crown-6 as an ionophore were investigated using this method. ${ }^{23}$ Different types of complexes were observed, which can be related to two possible mechanisms, i.e. the adsorption and/or extraction of an ion pair and the complexation formation at the interface. Further investigations were done on peptide-lipid complexation by reacting peptides present in an aqueous channel and phospholipids dissolved in an organic phase (1,2-dichloroethane). ${ }^{24}$

\section{Electrochemical aspects of laser desorption ionization}

For the electrochemical aspects of LDI, a lot of attention has been given to the photochemical aspects of the gas phase 
reactions, ${ }^{25}$ but very few studies have investigated the electrode reaction. Considering first the case of MALDI used in conjunction with a TOF detection unit, the process can be divided into two steps: (1) the photochemical step induced by a laser pulse where part of the analyte-matrix mixture is ablated to form a plume in which collision-induced charge transfer reactions take place and (2) the electrochemical step resulting from the application of a high voltage between the metallic plate and the gas phase to drive the ions to the detection unit. Often, the application of the voltage pulse is delayed with respect to the laser pulse. If we consider that an ion current results from the application of the voltage, an electrochemical reaction must occur on the metallic plate to ensure the continuity of the current in the loop illustrated in Figure 5(a).

It is clear that the electrochemical aspects of MALDI raise more questions than answers. To list just a few:

1. What is the nature of the electrode reaction on a metallic plate?

2. Can the matrix be considered as a solid electrolyte or even a proton carrier?

3. Can we control the electrode reaction to improve the MS analysis?

One way to answer some of these questions is to consider a matrix with well characterized photosensitizers such as porphyrin type molecules or matrix-free, semiconductorbased systems such as desorption/ionization on porous silicon (DIOS), ${ }^{5} \mathrm{TiO}_{2}{ }^{26}$ or $\mathrm{Ga}_{2} \mathrm{O}_{3}$ coated metallic plate. These materials have separated valence and conduction bands with a band gap matching the energy of UV light. Under UV laser irradiation during the LDI process, electrons can be ejected from the valence to the conduction band forming electronhole pairs. ${ }^{27}$ Some pairs recombine, either in the nanostructure or on its surface, releasing heat to the surrounding analytes, thereby favoring sample desorption. Otherwise, these electrons and holes can react with species present on the substrate and induce in-source redox reactions. In fact, a metallic plate coated with a mesoporous layer of nanoparticles, such as $\mathrm{TiO}_{2}$, resembles a dye-sensitized solar cell.
Upon light irradiation, two electrochemical processes can take place. Either a molecule ( $D=$ donor) is oxidized and, in this case, the electrons migrate through the mesoporous semiconductor layer to the metallic substrate, or a molecule ( $A$ = acceptor) is reduced, where electrons transfer from the metallic substrate into the porous structure of semiconductor. The productions of these redox reactions can still react with analytes to generate various ions, either directly on the surface of the semiconductor layer shortly after laser shot or in the plume during the ion extraction delay period. Parts of the ions can be extracted later by the applied electric field due to their charge type, while others would be forced back to the conductive sample substrate, Figure 5(b).

\section{In source oxidative peptide fragmentation on semiconductor substrate}

The semiconductor modified substrate can be obtained by sintering nanoparticles of $\mathrm{TiO}_{2}$ or $\mathrm{Ga}_{2} \mathrm{O}_{3}$ on a stainless steel target plate. Nanomaterials were separated to form stable suspension to be dropped onto the plate ${ }^{28}$ or made into a paste to be printed on the plate or a disposable layer, such as Al foil, ${ }^{29}$ and then heated to form a stable photosensitive layer. By controlling the photo-electrochemical reactions on the mesoporous $\mathrm{TiO}_{2}$, our groups have shown that it is possible to oxidize peptides to induce $a-x$ fragmentation rather than the classical $c-z$ fragmentation obtained through reductive pathways. ${ }^{28}$

Glucose was deposited, together with analytes, on a $\mathrm{TiO}_{2}$ modified target plate for the in-source $a-x$ decay. This novel dissociation pattern was first observed with angiotensin I and oxidized $\beta$-insulin. Here, glucose, a hole scavenger in classic photo-catalysis reactions, ${ }^{30}$ was proven to act as hole conductor, being oxidized on the surface of $\mathrm{TiO}_{2}$ nanoparticles by photo-initiated holes as radicals with the loss of one
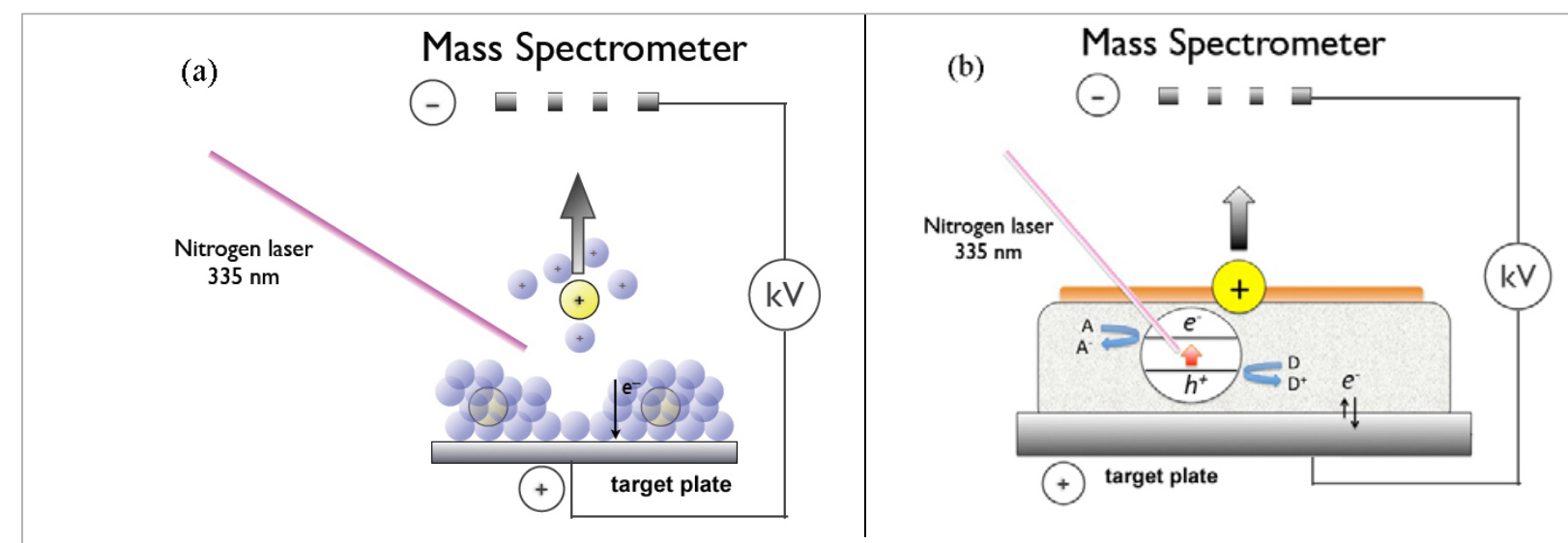

Figure 5. Schematic representation of (a) a MALDI process and (b) a semi-conductor-based LDI process and the inherent loop. 


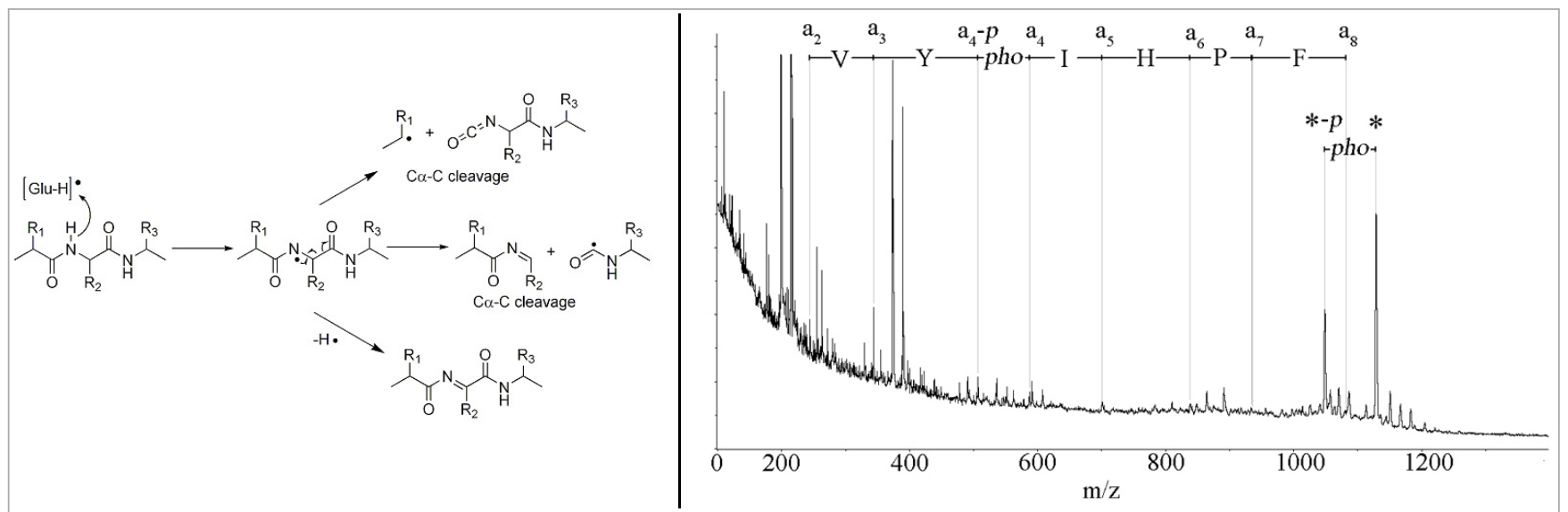

Figure 6. (a) Supposed mechanisms for the $\mathrm{C}_{\alpha}-\mathrm{C}$ bond cleavage during $\mathrm{TiO}_{2}$-induced photocatalytic peptide fragmentation; (b) Fragmentation result of phosphorylated angiotensin II ( $\sim 70 \mathrm{pmol}$, DRVpYIHPF) obtained on a $\mathrm{TiO}_{2}$ modified plate in the presence of glucose. *-tagged peaks correspond to parent ions and ax-tagged peaks correspond to a fragments, respectively.

hydrogen atom. The generated glucose radicals would still hold strong oxidative activity and then further react with each other or analytes, therefore realizing long-distance oxidation in the ionization plume. By snatching a hydrogen atom from the amide group of peptides, the glucose radical is reduced while the $\mathrm{C}_{\alpha}-\mathrm{C}$ bond is cleaved, Figure 6 (left). The present peptide fragmentation is quite different from traditional peptide in source decay (ISD) during matrix-assisted LDI, which is generally believed to be a reductive process induced by electrons, but rather similar to a so-called electrondetachment dissociation (EDD) reported by Zubarev et al. $^{31-33}$

Our recent research suggests that the oxidative peptide decay (OPD) obtained on a $\mathrm{TiO}_{2}$ substrate can be used for protein post-translational modification study. By using phosphorylated angiotensin II as an example, it is proven that the phospho-modification can be conserved during the OPD process and the modification sites can be easily read out from the fragmentation pattern, Figure 6 (right). On the mass spectrum, an a 4 ion, still holding the phospho-modification, is obtained as a fragment of the parent ion (*), while a dephosphorylated a 4 ion (a4-p), which could be a fragment of the dephosphorylated parent ion $\left({ }^{*}-p\right)$, is also found. Therefore, it is clear that the phosphorylation has happened on the $4^{\text {th }}$ amino acid residue of the peptides. Indeed, the dephosphorylated parent ion $\left({ }^{*}-p\right)$ can be generated from the high energy radiation or during ion flight in the reflection region of the TOF mass analyzer. The present method is promising for proteome research but still not efficient enough for complex sample analysis. As a first application, we have combined the OPD strategy with off-gel separation $^{34}$ for analyzing the phosphorylation of $\beta$-casein. In the future, it will be interesting to improve the energy conversion efficiency of semiconductor materials and to develop highperformance hole conductor reagents.

\section{In-source reduction for disulfide bond cleavage}

Although semiconductor materials such as $\mathrm{TiO}_{2}$ mainly show oxidative properties under light irradiation, they can also be used for photo-reduction in the presence of hole scavengers, where electrons are injected into the valence band of the semiconductor from these added species to eliminate holes and then the conduction band electrons can be largely preserved to further react with target molecules. By applying this principle in the LDI process, in-source reduction of samples was achieved. Specifically, D-glucose was used here as a hole scavenger, which has, indeed, previously proven to be efficient in classic photo catalysis research, ${ }^{30}$ while the disulfide bond in human insulin was selected as the target reductive group, ${ }^{27}$ Figure 7 (left). Human insulin is a protein with two chains, A-chain and B-chain, and three disulfide bonds. By cleaving these bonds, the intact human insulin molecule can be separated into two fragments of A-chain and $\mathrm{B}$-chain. Whereas the resolution is not good enough, it is obvious that the disulfide bond is cleaved during the LDI process on $\mathrm{TiO}_{2}$ substrate, Figure 7 (right), which is, indeed, a reductive process induced by electrons. ${ }^{35}$ In contrast, no cleavage was observed with the same laser intensity and other mass spectrometry conditions on metallic substrates with/without organic matrices. Because electrons are mainly present in the semiconductor conduction band and there is no electron conductor that can bring the electrons into the ionization plume, the disulfide bond cleavage here is believed to only happen on the surface of the substrate shortly after laser irradiation rather than in the following generated ionization plume. This is interesting from an electrochemistry viewpoint, but the extreme conditions may also lead to an inefficient reduction due to the very short reaction period. 

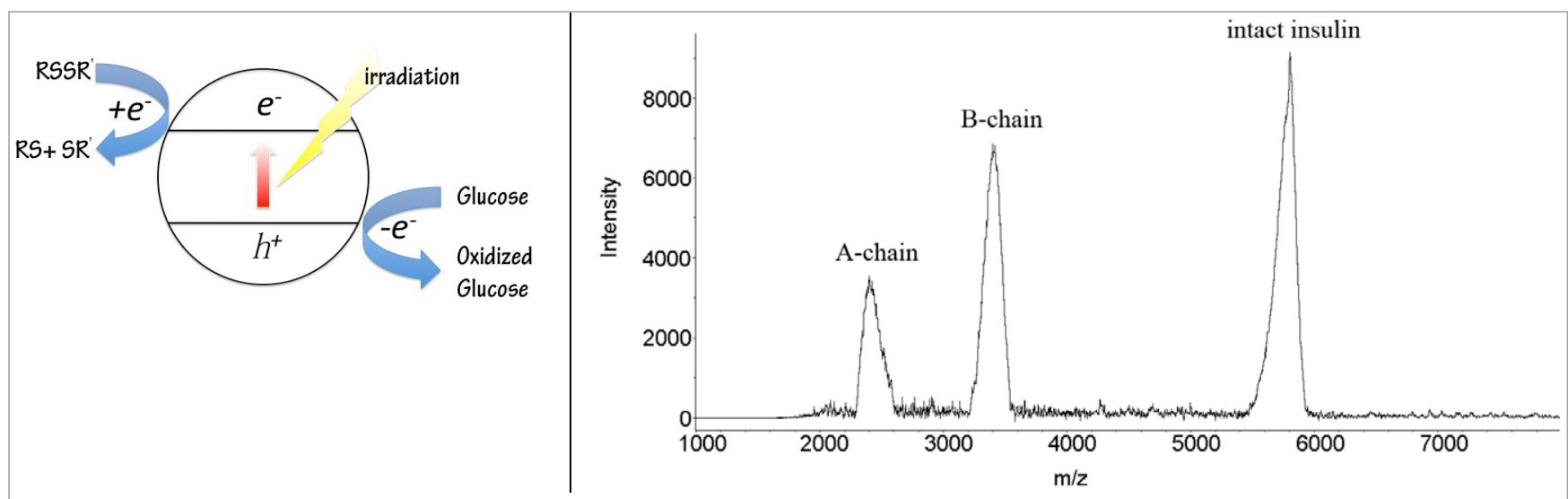

Figure 7. Photo-reduction of disulfide bond with the presence of electron donor D-glucose, and mass spectrum of products of this in-source reductive reaction from human insulin.

\section{In-source oxidation for peptide tagging on semiconductor substrates}

Similar to what has been described earlier, when adding hydroquinone to a peptidic sample, the on-plate oxidation of hydroquinone forms benzoquinone that can be used to tag cysteinecontaining peptides very efficiently. By depositing hydroquinone with analytes on the semiconductor-modified substrate, under laser irradiation, valence band holes in the substrate would easily oxidize hydroquinone to benzoquinone for following labelling reactions. This principle was first demonstrated on a $\mathrm{TiO}_{2}-$ modified substrate with standard cysteine-containing peptides ${ }^{36}$ and then applied in proteome research. Indeed, with this kind of on-line labeling method, information about cysteine content can be obtained as well as the molecule weight of a peptide in one experiment, therefore enhancing the identification accuracy during data profiling. ${ }^{37}$ Recently, a photosensitive $\beta-\mathrm{Ga}_{2} \mathrm{O}_{3}$ modified target plate has been designed for making the tagging more efficient and applied for specifically enhancing the identification of cysteine-containing proteins from protein mixtures.

\section{Conclusions}

All in all, this short review illustrates the electrochemical principles and applications of both electrospray and laser desorption ionization. It shows that a good control of these electrochemical and photo-electrochemical reactions can be put to good use to perform specific tasks such as tagging, or to oxidize or reduce in-situ target molecules.

\section{Acknowledgement}

The authors wish to thank the Swiss National Science Foundation for supporting the project "Analytical tools for proteome analysis and redoxomics (200020-127142)", and the National Science Foundation of China "NSFC 20925517".

\section{References}

1. M. Yamashita and J.B. Fenn, "Electrospray ion source. Another variation on the free-jet theme", J. Phys. Chem. 88, 4551-4459 (1984). doi: 10.1021/j150664a002

2. M. Karas, D. Bachmann, U. Bahr and F. Hillenkamp, "Matrix-assisted ultraviolet laser desorption of nonvolatile compounds", Int. J. Mass Spectrom. Ion Processes 78, 53-68 (1987). doi: 10.1016/0168-1176(87)87041-6

3. R. Aebersold and M. Mann, "Mass spectrometry-based proteomics", Nature 422, 198-207 (2003). doi: $\underline{10.1038 /}$ $\underline{\text { nature } 01511}$

4. M. Karas and R. Kruger, "Ion formation in MALDI: the cluster ionization mechanism", Chem. Rev. 103, 427-439 (2003). doi: 10.1021/cr010376a

5. J. Wei, J.M. Buriak and G. Siuzdak, "Desorptionionization mass spectrometry on porous silicon", Nature 399, 243-246 (1999). Doi: $10.1038 / 20400$

6. J.A. Nollet, Recherches sur les causes particulières des phénomènes électriques, 1ère edn. Chez les frères Guerin, Paris, France (1749).

7. J. Zeleny, "The electrical discharge from liquid points, and a hydrostatic method of measuring the electric intensity at their surfaces", Phys. Rev. 3, 69-91 (1914). doi: 10.1103/PhysRev.3.69

8. G.I. Taylor, "Disintegration of water drops in an electric field", Proc. R. Soc. London, Ser. A 280, 383-397 (1964). doi: $\underline{10.1098 / \text { rspa.1964.0151 }}$

9. C.F. Eyring, S.S. Mackeown and R.A. Millikan, "Fields currents from points", Phys. Rev. 31, 900-909 (1928). doi: 10.1103/PhysRev.31.900

10. R.A. Marcus, "On the theory of ion transfer rates across the interface of two immiscible liquids", J. Chem. Phys. 113, 1618-1629 (2000). doi: $10.1063 / 1.481950$ 
11. J.V. Iribarne and B.A. Thomson, "On the evaporation of small ions from charged droplets", J. Chem. Phys. 64, 2287-2294 (1976). doi: 10.1063/1.432536

12. C. Roussel, L. Dayon, N. Lion, T.C. Rohner, J. Josserand, J.S. Rossier, H. Jensen and H.H. Girault, "Generation of mass tags by the inherent electrochemistry of electrospray for protein mass spectrometry", J. Am. Soc. Mass Spectrom. 15, 1767-1779 (2004). doi: 10.1016/j. jasms.2004.08.006

13. T.C. Rohner, J.S. Rossier and H.H. Girault, "On-line electrochemical tagging of cysteines in proteins during nanospray", Electrochem. Commun. 4, 695-700 (2002). doi: $\underline{\text { 10.1016/S1388-2481(02)00426-5 }}$

14. L. Dayon, C. Roussel and H.H. Girault, "On-line electrochemical tagging of free cysteines in peptides during nanospray ionisation mass spectrometry: an overview", Chimia 58, 204-207 (2004). doi: $10.2533 / 000942904777678028$

15. L. Dayon, J. Josserand and H.H. Girault, “Electrochemical multi-tagging of cysteinyl peptides during microspray mass spectrometry: numerical simulation of consecutive reactions in a microchannel", Phys. Chem. Chem. Phys. 7, 4054-4060 (2005). doi: $10.1039 / \mathrm{b} 511334 \mathrm{~b}$

16. L. Dayon, C. Roussel, M. Prudent, N. Lion and H.H. Girault, "On-line counting of cysteine residues in peptides during electrospray ionization by electrogenerated tags and their application to protein identification", Electrophoresis 26, 238-247 (2005). doi: 10.1002/elps.200406144

17. M. Abonnenc, L. Dayon, B. Perruche, N. Lion and H.H. Girault, "Electrospray micromixer chip for on-line derivatization and kinetic studies", Anal. Chem. 80, 3372 3378 (2008). doi: 10.1021/ac800058h

18. L. Dayon, C. Roussel and H.H. Girault, "Probing cysteine reactivity in proteins by mass spectrometric EC-tagging", J. Proteome Res. 5, 793-800 (2006). doi: 10.1021/pr050365o

19. T.C. Rohner and H.H. Girault, "Study of peptide on-line complexation with transition-metal ions generated from sacrificial electrodes in thin-chip polymer microsprays", Rapid Commun. Mass Spectrom. 19, 1183-1190 (2005). doi: 10.1002/rcm.1899

20. M. Prudent, C. Roussel and H.H. Girault, "Electrochemical generation of Cu(I) complexes in aqueous solutions studied by on-line mass spectrometry", Electrochem. Commun. 9, 2067-2074 (2007). doi: 10.1016/i. elecom.2007.06.007

21. M. Prudent and H.H. Girault, "On-line electrogeneration of copper-peptide complexes in microspray mass spectrometry", J. Am. Soc. Mass Spectrom. 19, 560-568 (2008). doi: 10.1016/i.jasms.2008.01.008

22. M. Prudent, J.S. Rossier, N. Lion and H.H. Girault, "Microfabricated dual sprayer for on-line mass tagging of phosphopeptides", Anal. Chem. 80, 2531-2538 (2008). doi: $\underline{10.1021 / a c 7025139}$
23. M. Prudent, M.A. Méndez and H.H. Girault, “Biphasic electrospray ionization for the study of interfacial complexes", Anal. Sci. 24, 1399-1404 (2008). doi: $10.2116 /$ analsci.24.1399

24. M.A. Méndez, M. Prudent, B. Su and H.H. Girault, "Peptide-phospholipid complex formation at liquid-liquid interfaces", Anal. Chem. 80, 9499-9507 (2008). doi: $\underline{10.1021 / a c 801651 f}$

25. R. Knochenmuss, A. Stortelder, K. Breuker and R. Zenobi, "Secondary ion-molecule reactions in matrix-assisted laser desorption/ionization", J. Mass Spectrom. 35, 1237-1245 (2000). doi: 10.1002/1096-9888(200011)35:11<1237::AIDJMS74>3.0.CO;2-0

26. C.T. Chen and Y.C. Chen, "Desorption/ionization mass spectrometry on nanocrystalline titania sol-geldeposited films", Rapid Commun. Mass Spectrom. 18, 1956-1964 (2004). doi: 10.1002/rcm.1572

27. L. Qiao, H.Y. Bi, J.M. Busnel, B.H. Liu and H.H. Girault, "In-source photocatalytic reduction of disulfide bonds during laser desorption ionization”, Chem. Commun. 47, 6357-6359 (2008). doi: 10.1039/b813283f

28. L. Qiao, H.Y. Bi, J.M. Busnel, J. Waser, P.Y. Yang, H.H. Girault and B.H. Liu, "Photocatalytic redox reactions for in-source peptide fragmentation”, Chem. Eur. J. 15, 6711-6717 (2009). doi: 10.1002/chem.200802229

29. H.Y. Bi, L. Qiao, J.M. Busnel, V. Devaud, B.H. Liu and H.H. Girault, " $\mathrm{TiO}_{2}$ printed aluminum foil: single-use film for a laser desorption/ionization target plate", Anal. Chem. 81, 1177-1183 (2009). doi: 10.1021/ac8024448

30. I.A. Shkrob, M.C. Sauer and D. Gosztola, “ Efficient, rapid photooxidation of chemisorbed polyhydroxyl alcohols and carbohydrates by $\mathrm{TiO}_{2}$ nanoparticles in an aqueous solution", J. Phys. Chem. B 108, 12512-12517

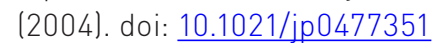

31. F. Kjeldsen, O.A. Silivra, I.A. Ivonin, K.F. Haselmann, M. Gorshkov and R.A. Zubarev, " $\mathrm{C} \alpha-C$ backbone fragmentation dominates in electron detachment dissociation of gas-phase polypeptide polyanions", Chem. Eur. J. 11, 1803-1812 (2005). doi: 10.1002/chem.200400806

32. K.F. Haselmann, B.A. Budnik, F. Kjeldsen, M.L. Nielsen, J.V. Olsen and R.A. Zubarev, "Electronic excitation gives informative fragmentation of polypeptide cations and anions", Eur. J. Mass Spectrom. 8, 117-121 (2002). doi: 10.1255/ejms. 479

33. B.A. Budnik, K.F. Haselmann and R.A. Zubarev, "Electron detachment dissociation of peptide di-anions: an electron-hole recombination phenomenon", Chem. Phys. Lett. 342, 299-302 (2001). doi: 10.1016/S00092614(01)00501-2

34. A. Ros, M. Faupel, H. Mees, J. van Oostrum, R. Ferrigno, F. Reymond, P. Michel, J.S. Rossier and H.H. Girault, "Protein purification by off-gel electrophoresis", Proteomics 2, 151-156 (2002). doi: 10.1002/1615-9861(200202)2:2<151::AIDPROT151>3.0.C0;2-9 
35. Y. Fukuyama, S. Iwamoto and K. Tanaka, "Rapid sequencing and disulfide mapping of peptides containing disulfide bonds by using 1,5-diaminonaphthalene as a reductive matrix", J. Mass Spectrom. 41, 191-201 (2006). doi: 10.1002/jms.977

36. L. Qiao, C. Roussel, J.J. Wan, J. Kong, P.Y. Yang, H.H. Girault and B.H. Liu, "MALDI in-source photooxidation reactions for online peptide tagging", Angew. Chemie Int Ed. 47, 2646-2648 (2008). doi: 10.1002/anie.200703876

37. S. Sechi and B.T. Chait, "Modification of cysteine residues by alkylation. a tool in peptide mapping and protein identification", Anal. Chem. 70, 5150-5158 (1998). doi: $\underline{10.1021 / a c 9806005}$ 